\title{
Intelligent Microclimate Control System Based on loT
}

\author{
Aigerim Bakatkaliyevna Altayeva, Batyrkhan Sultanovich Omarov, and Young \\ Im Cho \\ Department of Computer Engineering, Gachon University, Seongnam, Korea
}

\section{]jfis}

\begin{abstract}
The present research paper is devoted to solving an urgent problem, i.e., the energy saving and energy efficiency of buildings. A rapid settlement method and experimental control of the energy conservation based on the specific characteristics of the thermal energy consumption for the heating and ventilation of the buildings, and as well as the rapid development of wireless sensor networks, can be used in a variety of monitoring parameters in our daily lives. Today's world has become quite advanced with smart appliances and devices such as laptops, tablets, TVs, and smartphones with various functions, and their use has increased significantly in our day-to-day lives. In this case, the most important role is played by a wireless sensor network with its development and use in heterogeneous areas and in several different contexts. The fields of home automation, process management, and health management systems make extensive use of wireless sensor networks. In this paper, we explore the main factors of the microclimate in an indoor environment. We control the temperature humidity, and other factors remotely using sensors and Internet-of-Things technologies.
\end{abstract}

Keywords: IOT, Smart indoor environment, Heating, Ventilation, Air conditioning system, Sensors

\section{Introduction}

The microclimate in a room is a fundamental factor of a healthy life, and is determined by many physical aspects, such as the temperature, humidity, concentration of harmful substances, and speed of moving air (wind). These factors determine whether a person feels a "thermal

Received: Dec. 12, 2016

Revised : Dec. 13, 2016

Accepted: Dec. 14, 2016

Correspondence to: Young Im Cho (yicho@gachon.ac.kr)

(C)The Korean Institute of Intelligent Systems

(c) This is an Open Access article distributed under the terms of the Creative Commons Attribution Non-Commercial License (http://creativecommons.org/licenses/ by-nc/3.0// which permits unrestricted noncommercial use, distribution, and reproduction in any medium, provided the original work is properly cited. comfort," i.e., a sense of hot or cold. Comfort occurs when the body does not require a thermoregulatory mechanism, i.e., no tremors or sweating occurs, and the blood flow can maintain a constant speed in the peripheral organs. This condition corresponds to a thermal neutral zone [1]. The four physical factors are to some extent interchangeable with regard to the feeling of comfort and thermoregulation needs. In other words, the feeling of cold caused by a low-temperature air supply can be reduced corresponding to the temperature radiation of warm walls. If the atmosphere feels stuffy, the corresponding feeling can be alleviated through a reduction in the temperature or air humidity. Finally, if the radiation temperature is low (cold walls), an increase in temperature is required.

Thermal comfort is defined as a condition in which satisfaction with the ambient temperature is expressed. To this end, the physical and physical and biological factors are affected.

It is difficult to describe the general conditions that must be met for thermal comfort because 
various operating conditions occur under different situations. Different conditions may be required even for the same work when carried out by different people. The human body is constantly releasing heat depending on its physical activity. As an example, a calmly sleeping adult allocates an average of about $80 \mathrm{~W}$, and during great physical effort allocates about $300 \mathrm{~W}$. The technical standards for the temperature conditions required for comfort are not applicable to all regions owing to the different climatic conditions and clothing traditions.

The temperature and humidity in the room are the most important parameters determining the state of comfort.

\section{Climate Control Organization}

To maintain a given level of microclimate and indoor air quality, it is necessary to develop a regulatory system with economical consumption and power for the specified operating conditions.

HVAC systems consist of a number of subsystems, each of which may have nonlinear characteristics that vary with time. Moreover, the parameters of a dynamic state usually vary depending on the weather and disturbances. The use of conventional management schemes for such technology and management systems with a large number of adjustable parameters has been shown to be ineffective.

To control the HVAC system in a facility, a classical GDSregulation system can be used, although the HVAC system may be affected when the object parameters are changed [2]. The introduction of additional controls has certain disadvantages, such as an inconvenient parameter setup, interference, and slight deviations from the set values.

Fuzzy logic control allows many regulatory shortcomings to be eliminated, and is based on the use of standard transfer functions. A control algorithm based on fuzzy logic can be obtained from an analysis of the process used to maintain a given indoor environment, and optimized by changing the operating mode. Through this system, many advantages including a powerful anti-interference, more rapid actions, and high operation reliability are achieved.

Considering both its advantages and disadvantages, fuzzylogic based control is at least an adequate alternative solution. One of the main advantages of such systems is an easy migration without changing the structure of the control circuit. Compared with traditional automatic control methods, the application of fuzzy systems allows one to quickly analyze the data and obtain the results with a high level of accuracy. A characteristic feature of methods solving the problems of fuzzy logic is the availability of a set of rules consisting of a set of conditions and conclusions. Fuzzy control methods are now one of the most important steps in the development of smart technologies for creating a highly organized management system.

A special quality-control system, in particular, has low sensitivity to changes in the control parameters of an object. The synthesis of control systems with fuzzy logic in the application of modern hardware and software support is simpler than the synthesis of traditional management systems.

However, this is usually insufficient to control the air temperature mode.

The control system consists of a cooler (1), a heater (2), a humidifier (3), a regulated fan (4) that provides an air supply, a fuzzy controller (5), outside temperature sensors (6), an outdoor humidity sensor (7), inside temperature sensors (8), indoor humidity sensors (9), control zones (10), and air channel systems (11).

This system was designed for climate control of a building, and for increasing or lowering the temperature and relative humidity.

\subsection{Temperature}

Adjusting the air temperature in a "smart house" system is carried out with the help of special sensors placed inside and outside. They measure the temperature, and if it does not meet the specified criteria, send a signal to the main control panel. Next, a number of measures to achieve the optimum performance are applied, including automatic temperature control, which maintains the set temperature of the room. In addition, the available range of temperatures is zero to $125^{\circ} \mathrm{C}$.

A condensed electric heating system that can be programmed on a weekly or monthly cycle of heating is used. Temperature control in a room is somewhat more complicated. A thermostat should not only be able to control the heating of the air and the fan speed, but also the optimum temperature control algorithm for the air-cooling of the air-conditioning system [3].

The task is to determine the temperature corresponding to the level of control of the digital-to-analog converter regulator, the input variables of which are as follows: $e$, which is the difference between the desired and actual temperature in Celsius, and $\Delta e$, the first derivative of the temperature change during the computing cycle, in ${ }^{\circ} \mathrm{C} / \mathrm{min}$.

$$
\Delta e=T_{\text {given }}(t)-T_{\text {current }}(t),
$$

where $T_{\text {given }}(t)$ and $T_{\text {current }}(t)$ are the given and current tem- 
peratures in ${ }^{\circ} \mathrm{C} / \mathrm{min}$.

Naturally, the greater the temperature difference at a particular moment, the greater the required increase in cooling or heating rate. The rate of change of temperature is indicated as

$$
\Delta e=\frac{e\left(t_{1}\right)-e\left(t_{2}\right)}{t_{1}-t_{2}} .
$$

As the temperature of the room approaches the set point, the rate of change of the temperature will decrease, for example, during the air-conditioning cooling step.

We define the linguistic variables for fuzzy $e$ and $\Delta e$ fuzzy sets using the appropriate identification for membership functions $\mu(e)$ and $\mu(\Delta e)$.

We construct two membership functions. In one, the argument is the temperature difference (e) (Figure 1(a)), and in the second, the argument is the rate of temperature change (Figure 1(b)). The first function has a temperature range of -6 to $8^{\circ} \mathrm{C}$, and the second function has a temperature range of -6 to $8^{\circ} \mathrm{C} / \mathrm{min}$. For $\mu(e)$ and $\mu(\Delta e)$ (Figure 1), the identifiers are defined as: the deviation is strongly positive (PB), the deviation is moderately positive (PM), the deviation is slightly positive (PS), the deviation is zero (Z), the deviation is slightly negative (NS), the deviation is moderately negative (NM), and the deviation is strongly negative (NB).

The result from the joint effect of the two membership functions of the output parameter value is determined through the corresponding program embedded in the logic device.

With the help of the membership function (Figure 1(a)), the desired mode of operation of the heating and cooling systems is given. The fuzzy variables are the identifiers, "strong cooling" (C3), "moderate cooling" (C2), "slight cooling" (C1), "unchanged" (NO), "heating 1" (H1), and "heating 2" (H2), and are calculated using the fan speed based on a predetermined rule base (Figure 1(b)). The fuzzy variables corresponding to the fan speed identifiers are "high" (fast), "normal" (moderate), "low" (low), and "zero" (Z).

The function accessory output (Figure 2) shows the rule processing, and sums the response signals to generate an output command. The selected functions in this paper supply an output consisting of the two heating levels ( $\mathrm{H} 1$ and $\mathrm{H} 2)$, three cooling levels ( $\mathrm{C} 1, \mathrm{C} 2$, and $\mathrm{C} 3$ ), and normal level (NO), and it is possible to imagine several different levels of additional heating or cooling, with a heating value of greater than $\mathrm{H} 1$ or $\mathrm{H} 2$; in addition, the value of $\mathrm{C} 3$ is greater than that of $\mathrm{C} 2$ and $\mathrm{C} 1$.

The rules are listed in Table 1, and are shown as the applied linguistic variables obtained by summing the fuzzification for a

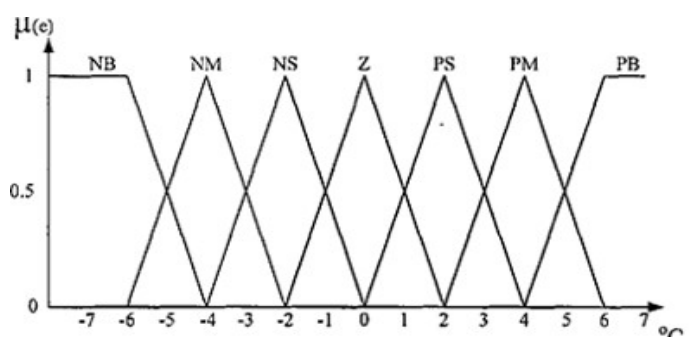

(a)

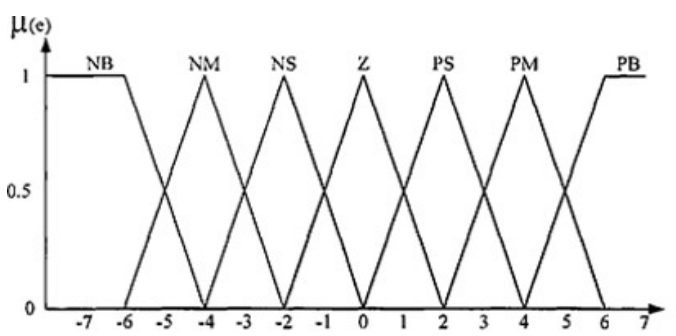

(b)

Figure 1. Linguistic membership functions for the input parameters.
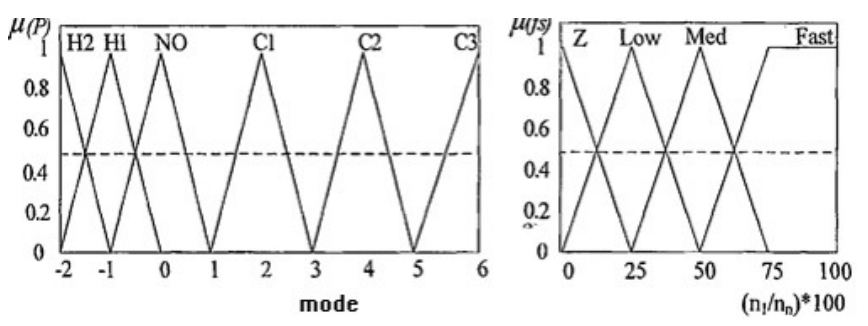

Figure 2. Linguistic membership functions for output parameters.

response using operator intuition [4]. When connected to the output membership function and the corresponding de- fuzzification, a clear signal to the control action is obtained. In this case, the control signal is determined based on the level of heating or cooling from the data $[-2,-1,0,1,2,3 \ldots]$.

The relationships between the input and output are listed in the table of fuzzy rules (Table 1). Each entry corresponds to a fuzzy rule. For example, if the current internal temperature is $30^{\circ} \mathrm{C}$ and the preset temperature is $24^{\circ} \mathrm{C}$, which means $e=$ $-6^{\circ} \mathrm{C}(\mathrm{NB})$, and if the deviation is strongly positive, then the room is cooled to level C3, the fan speed is "very high" (fast), and the heating rate will be equal to zero. The logical notation is as follows: if $e=\mathrm{NB}, \mathrm{C} 3$ is used and the fan speed is fast.

Communication between the input and output data is achieved through a distinct linguistic transformation of the input data. The input membership functions can be found through the implication and a summation using the rule base and defuzzification of linguistic output in numerical values (degree of heating or 
Table 1. Fuzzy control terms

\begin{tabular}{|c|c|c|c|c|c|c|c|c|}
\hline & & & & ape & 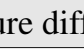 & erer & (e) & \\
\hline & & NB & NM & NS & Z & PS & PM & PB \\
\hline & NB & C3 & C3 & $\mathrm{C} 2$ & $\mathrm{C} 1$ & NO & NO & H1 \\
\hline & & Fast & Fast & Med & Slow & $\mathrm{Z}$ & 2 & Med \\
\hline & NM & C3 & $\mathrm{C} 2$ & $\mathrm{C} 2$ & $\mathrm{C} 1$ & NO & NO & H1 \\
\hline & & Fast & Med & Med & Slow & Z & Z & Med \\
\hline $\begin{array}{l}\Delta e \\
\text { Rate }\end{array}$ & NS & $\mathrm{C} 3$ & $\mathrm{C} 2$ & $\mathrm{C} 1$ & $\mathrm{C} 1$ & NO & NO & H1 \\
\hline 4 & & Fast & Med & Slow & Slow & $\mathrm{Z}$ & $\mathrm{Z}$ & Med \\
\hline tem- & Z & $\mathrm{C} 2$ & $\mathrm{C} 1$ & $\mathrm{C} 1$ & NO & NO & H1 & H1 \\
\hline & & Med & Slow & Slow & Z & Z & Med & Med \\
\hline change & PS & $\mathrm{C} 1$ & $\mathrm{C} 1$ & NO & NO & H1 & H1 & $\mathrm{H} 2$ \\
\hline & & Slow & Slow & Z & Z & Med & Med & Fast \\
\hline & PM & $\mathrm{C} 1$ & C1 & $\mathrm{NO}$ & NO & H1 & $\mathrm{H} 2$ & $\mathrm{H} 2$ \\
\hline & & Slow & Slow & Z & Z & Med & Fast & Fast \\
\hline & PB & $\mathrm{C} 1$ & $\mathrm{C} 1$ & $\mathrm{NO}$ & NO & $\mathrm{H} 2$ & $\mathrm{H} 2$ & $\mathrm{H} 2$ \\
\hline & & Slow & Slow & $\mathrm{Z}$ & Z & Fast & Fast & Fast \\
\hline$e$ & & & + ver & cold & & & cond & ioner \\
\hline & & & $-v e$ & hot & & & & \\
\hline$\Delta e$ & & $+\mathrm{l}$ & eat cor & sumpt & & & vent & lator \\
\hline & & 1. & & & $t$ & & & \\
\hline
\end{tabular}

cooling). Negative numbers refer to the degree of heating, and positive values refer to the degree of cooling. The output value of the controller is a discrete number of $[-2,1,0,1,2,3 \ldots]$.

\subsection{Ventilation}

Fresh air is one of the main advantages of a house. However, the hope that fresh air would be in the room itself or exclusively through air vents, a big mistake. This is particularly true of premises with a complex geometry and functionality.

The main feature of an intelligent ventilation control system is the ability to analyze the need for a ventilation of the premises. Each room in a house or apartment selects a comfortable atmosphere. In addition, "intelligent" ventilation only works when needed. For example, the system turns on during the evening to allow cool air to come into the bedroom overnight. However, if the residents are at home, the system stops.

"Intelligent" ventilation is not only able to bring fresh air into the home, but it also ensures that the air is as clean as possible. Such a system is controlled based on the schedule, e.g., the ventilation is switched on automatically at a certain time, for example, one hour before bedtime, or smoke detectors are triggered in the case of high concentrations of carbon dioxide [5]. Under the optimum air parameters, the system will operate in economy mode. Intelligent ventilation, which ensures that a home has a constant influx of fresh air, removes contaminated air, does not allow street noise to enter, and creates a healthy climate, all without the user's participation or control.

\subsection{Humidity}

The optimal humidity for normal human life is considered to be 40\%-60\%. Deviations in either direction can lead to negative consequences. In particular, overly dry air reduces the body's resistance to viral diseases, and too much humidity in a room promotes the growth of mold and mildew, and causes a variety of allergic reactions [5].

Achieving the optimum humidity level is not so simple. A normal air conditioner is not able to cope with such tasks. To help with this situation, come special humidifiers and dehumidifiers that can be successfully used in a "smart home" system have been developed. Special sensors monitoring the humidity levels send signals to the control unit, which automatically adjusts the performance by dehumidifying or humidifying the air.

\subsection{Air Conditioning}

Air conditioners are used in many apartments and houses. Current models are characterized by a high functionality and a variety of designs. However, such equipment is not always used effectively [6]. To solve this problem, a "smart home" system can be used that will set the operation of such equipment, namely, automatically turning on or off all air conditioners installed in different rooms, or each unit separately, to regulate the temperature, intensity, and air flow direction.

\section{Architecture of the System}

A base station located indoors is tied to a personal profile in the cloud. The base station processor determines what to do with a breather, air conditioner, and other actuators. Teams are based on user preferences. Peripheral sensors are located in other rooms, and communicate with the base station through an RFchannel. The communication base station is built with actuators, which are also on an RF-channel. Communication is made with third-party devices using an IR module that can be purchased additionally. A IR module is also a peripheral device that communicates with the base station using the same RF-module. The 


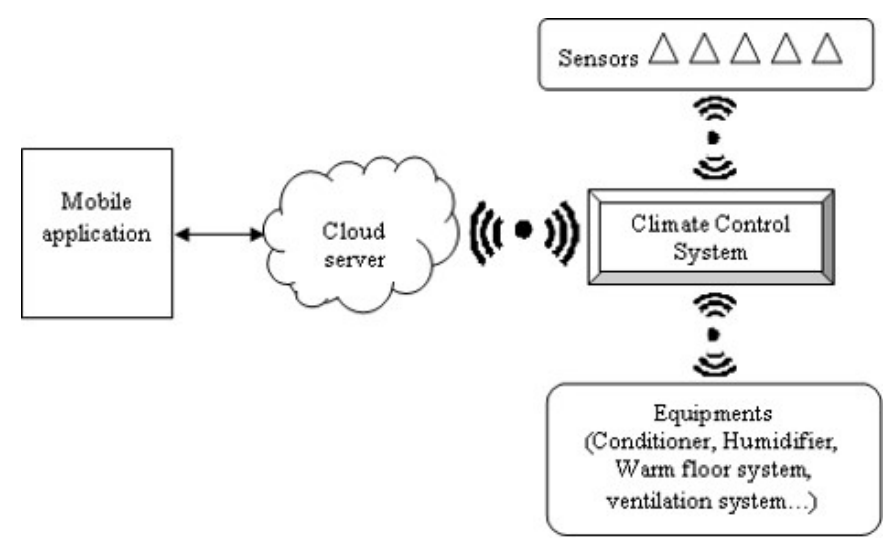

Figure 3. Architecture of the proposed system.

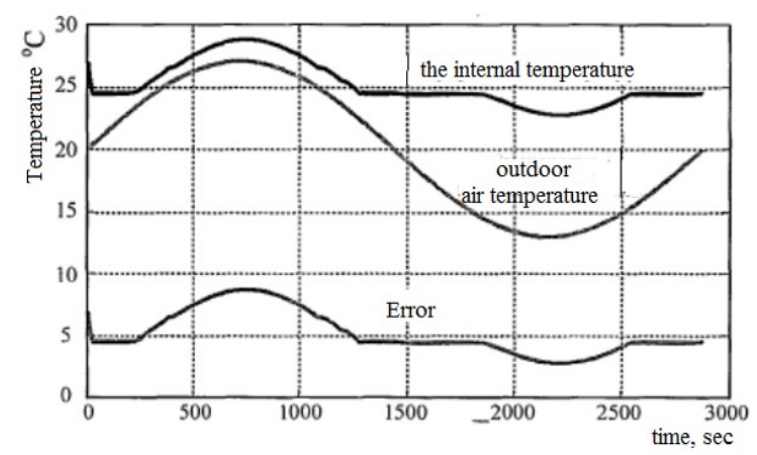

Figure 4. Graph of changes in the internal and external temperatures of the building climate control system.

backend and frontend connect through any Internet communication channel using Wi-Fi, 3G, or other networking type. Figure 3 illustrates the architecture of the proposed system.

In a cloud-based server, all parameters regarding the microclimate, information on the system architecture, and the state of all actuators are given through a personal user profile. Big data are immediately analyzed, and the correlation for teaching the computational algorithms is tracked.

Control actuators on a smartphone are applied indirectly through the backend along the entire chain: frontend - Internet backend - Internet - Wi-Fi - RF.

To demonstrate the fuzzy controller, we used a dynamic building model [6]. Schedule changes in the internal and external temperatures of the building climate control system and errors are shown in Figure 4.

The transient characteristics of the system with a stepwise temperature change (one step $-6^{\circ} \mathrm{C}$ ) within a given range (target temperature controller, $24-30^{\circ} \mathrm{C}$ ) for a change in time of 500-second steps are shown in Figure 5. The settling time is relatively small $\left( \pm 10 \%\right.$ of the final value of $\left.30^{\circ} \mathrm{C}\right)$. When the

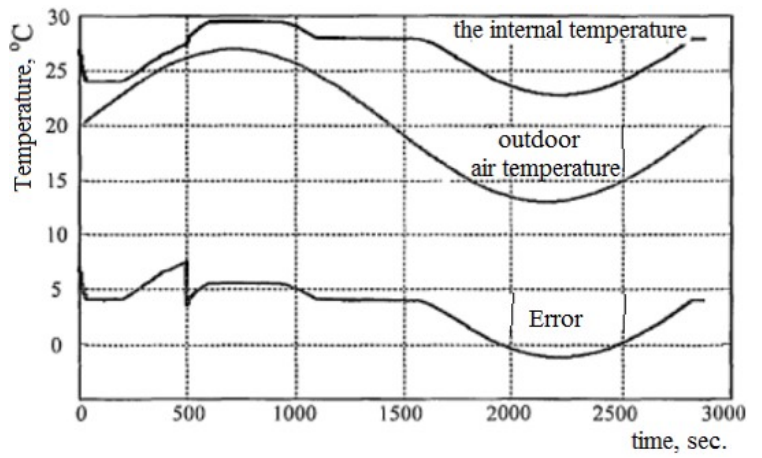

Figure 5. The step-wise response of the system to a step-wise change in the set temperature.

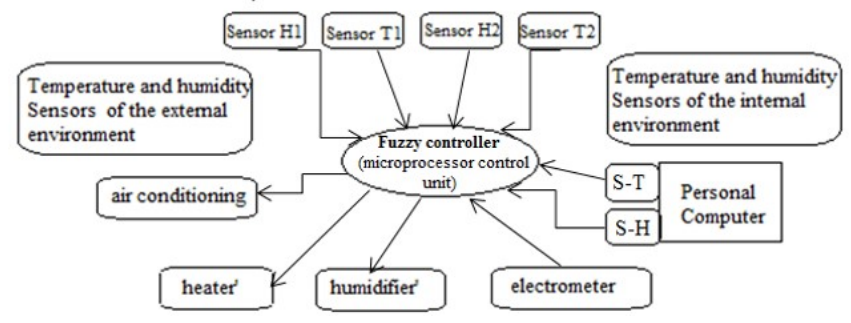

Figure 6. Functional diagram of the experimental setup for climate control system.

outside temperature falls to $30^{\circ} \mathrm{C}$, a static error begins to appear. These relationships show how well the system should track the target temperature.

Single-phase electricity meters are designed for direct connection of AC electrical energy at 50 to $60 \mathrm{~Hz}$. A counter may also be used in automated systems control and in the distribution of electrical energy. Using all of these devices and described principles, a prototype of the climate control system was developed. Figure 6 shows a functional diagram of the experimental setup used for the climate control system.

The installation allows labs to study the regulation of fuzzy logic. The composition of the stand is as follows:

- physical model of the premises;

- fuzzy controller (microprocessor control unit);

- sensors for external and internal temperature and humidity conditions;

- heater;

- air conditioning unit;

- steam humidifier; and

- personal computer.

The physical model is implemented as a space partially isolated from the surrounding room environment $(6 \mathrm{~m} \times 3 \mathrm{~m} \times$ $2.5 \mathrm{~m}$ room size), and includes temperature sensors, humidity 
sensors and actuators, i.e., a heater (with two operating modes, the first at 1,000 W, and the second at 2,000 W), a steam humidifier, and an air conditioner with four modes of $500 \mathrm{~W}$ each, and acts as a ventilation system. The sensors and actuators are spaced in the physical model to ensure the least interference in the control loop when the system is operating.

\section{Calculations}

For each zone, the indoor environment is characterized by high occupancy rates and the movement of people, which affect the design of the microclimate parameters. Of particular importance is the level of humidity regulation for an indoor environment.

Based on the above, and taking into account the purpose of separate rooms designated for different types of activities, one can determine the minimum design parameters of the volume flow rate for individual rooms. The calculations should take into account the amount of air water vapor produced by sweating, as well as the number of people. Calculating the volume flow based solely on the required air exchange data (usually from 60 to $120 \mathrm{~m}^{2} / \mathrm{h}$ per person) is insufficient because a correction for the post-dripping and heat demand is necessary. After determining the total volume of water generation in the room $\left(Q_{m v}\right)$, the volumetric flow rate of air required to remove moisture from the air is determined based on the difference between the absolute humidity and the internal supply air, and is calculated as follows:

$$
\begin{array}{r}
Q_{m a}=\frac{A_{m u}}{x_{a}}-x_{m}, \text { and } \\
V-a=\frac{A_{m a}}{p_{a}} .
\end{array}
$$

The amount of air needed to neutralize the physical heat load (qs) is determined based on the difference in temperature between the inside of the room and the fresh air outside, and is calculated as follows:

$$
V_{a}=\frac{Q_{S}(\text { thermalloading })}{0.34 \cdot \Delta t} .
$$

It should be noted that, during physical exercise, the human body consumes appreciable amounts of oxygen from the air. Thus, the more intense the activity intended for the room, the more important it is to provide the required air exchange, regardless of how much the actual heat and humidity of the room satisfy the regulatory requirements or the calculated data. To provide the necessary comfort, the rooms in operation should be continuously fed with a constant influx of outside air.

Based on a comparison of the internal and external factors, a microcontroller calculates the target temperature in the building every minute. In this calculation, not only are data obtained from the sensors for a particular moment used, but so is the background state of the building's microclimate. The features of this algorithm are as follows:

- the ability to maintain the desired indoor climate;

- coordinated management of the entire climate control system, which allows the optimum ratio of heat saving of the resources and the quality of the climate control to be achieved; and

- monitoring of the actual thermal capacity heating and ventilation circuits, as well as the redistribution of heat flows when the circuits are operating improperly.

The software system allows one to archive and graphically display all set and measured climate parameters in real time, and to calculate them in accordance with predetermined algorithm control actions. Every minute the controller transmits information on 28 measured and calculated values. The program provides real-time analysis of the systems management and delivery of the text mode and diagnostic alarms. The climate change parameters, control strategy, setting adjustments of the algorithm, calibration coefficients of all measurement sensors, and the limit values of the measured quantities above, which are issued by the appropriate diagnostic and alarm messages, are all given in tabular form. When transferring the set values and climate parameters that affect the management to the master controller, the data entered are automatically controlled in terms of admissibility, and thus the transmission of incorrect values is avoided.

The software system allows the dynamics of the climate during the day to be set in tabular form and graphically displayed according to the specified requirements.

The thermal processes of a heated space are a series of interconnected heat exchange sub-processes, and heat transfer between the elements of the system include the indoor air, building fence, internal contents, heaters, and ventilation system. These elements interact with each other and the environment through heat and mass transfer. For the building fence, air and heat exchange element boundaries are achieved through convection and radiation, respectively. In addition, the heat is 
transferred through convection from the radiators to the internal air space. The heat from the inner to outer surface of an enclosure is passed through a heat transfer. In addition, energy partitioning is carried out based on the heat transfer processes through the facilities. In addition to the thermal processes, mass transfer processes should be considered owing to the need for room ventilation.

Thus, a heated building can be displayed on the graph $G=$ $\{X, U\}$, where $X$ is the set of vertices, each of which is $x_{k}$ $(k=1, n)$, which corresponds to the internal air space of the $k$-th building, and $U$ is a plurality of edges, each of which corresponds to the heat or material flow between adjacent rooms. We should also highlight the vertex of $x_{0}$, which represents the ambient air.

Each vertex of the graph $x_{k}$ may have thermodynamic parameters of air, $V_{k}$ and $T_{k}$, which are the volume and temperature, respectively. For $U_{i j}$, each edge corresponds to a vector $\left(H_{i j}\right.$ $, c_{i j}, \rho_{i j}, F_{i j}, \lambda_{i i}$, where $H_{i}$ is the thickness, $F_{i j}$ is the surface, $i j c$ is the specific heat, $\rho_{i j}$ is the density, and $\lambda_{i j}$ is the fencing of thermal conductivity between the elements of the structures of $i$ and $j$. In addition to these system parameters defining purely thermal processes, one must also enter the parameters responsible for the mass transfer associated with the ventilation of the premises and the possible exchange of air masses between rooms. This parameter can be viewed obliquely in symmetric matrix $G_{i j}(i, j=0, n)$ for the weight (or volume) of air flow between the $i$-th and $j$-th elements.

Each edge of the structural graph $u_{i i}$ symbolizes the heat flow $j$ of $q_{i i}$ for element $x_{i}$ to element $x_{j}$. In turn, the heat flow $q_{i i}$ is determined by the laws of heat transfer in accordance with which, within the fence between the $i$-th and $j$-th rooms, a $T_{i i}(x, t)$ unsteady temperature field is formed, where $x \in$ $\left[0, H_{i i}\right]$ is the linear coordinate perpendicular to the surfaces of the fence. The specified temperature field described by the differential equation of heat conduction is

$$
\frac{\partial T_{i j}}{\partial t}=a_{i j} \frac{\partial^{2} T_{i j}}{\partial x^{2}},
$$

where $a_{i j}=\frac{\lambda_{i j}}{c_{i j} \rho_{i j}}$ the coefficient of thermal conductivity.

At the boundaries of the fence, $x=0$ and $x=H_{i j}$, the temperature satisfies the third type of boundary conditions, whereby the convective heat flux from the air to the fence is the heat flow inside the enclosure, i.e.,

$$
\alpha\left(T_{i}-T_{i j}(x, t)\right)_{x=0}=-\lambda_{i j}\left(\frac{\partial T_{i j}(x, t)}{\partial x}\right),
$$

$$
\alpha\left(T_{i j}(x, t)-T_{j}\right)_{x=H_{i j}}=-\lambda_{i j}\left(\frac{\partial T_{i j}(x, t)}{\partial x}\right)_{x=H_{i j}},
$$

where $\alpha$ is the convective heat transfer coefficient.

In turn, the temperature $T_{i}$ of each structural element can be determined based on the heat balance equation, which has the following form :

$$
\begin{aligned}
c_{v} \rho_{B} V_{i} \frac{d T_{i}}{d t}= & \alpha \sum_{\substack{j=0 \\
j \neq 0}}^{n} F_{i j}\left(T_{i j}(0, t)-T_{i}\right) \\
& +c_{p} \sum_{\substack{j=0 \\
j \neq 0}}^{n} G_{i j}\left(T_{j}-T_{i}\right)+\alpha F_{a i}\left(T_{a i}-T_{i}\right) \\
& +Q_{i}(t)
\end{aligned}
$$

where $c_{v}$ and $c_{p}$ are the specific isochoric and isobaric heat capacities of air, respectively; $\rho_{B}$ is the density of air; $F_{a i}$ and $T_{a i}$ are the surface area and temperature of the internal battery in the $i$-th room, respectively; and $Q_{i}(t)$ is the heat flux from the heat supplied from the heaters..

Eqs. (6) and (9) of the system need to be supplemented through a system of differential equations describing the thermal processes in the internal battery, which consists of various production equipment, furniture, and other space-filling objects:

$$
c_{i} \frac{d T_{a i}}{d t}=\alpha F_{a i}\left(T_{i}-T_{a i}\right),
$$

where $c_{i}$ is the capacity of the heat accumulators.

Thus, the mathematical model of the thermal processes of a building to be heated is a system of $n(n-1) / 2$ differential equations in the partial derivatives of form (6) with boundary conditions (7) and (8), and a system of $2 n$ ordinary differential thermal balance equations (9) and (10). For integration of the system of (6), (9), and (10) it is necessary to set the initial conditions for the temperature $T_{i}(i=1, n)$, the initial distribution of temperature fields $T_{i j}(x, 0)$, and the laws of the heat $Q_{i}(t)$ and mass transfer $G_{i j}(t)$. The results of such integration will be function $T_{i}(t)$, i.e., changes in air temperature.

\section{Conclusion}

This paper illustrated that the optimal levels of temperature, humidity, and air cleanliness in an apartment or house can be easily achieved by integrating all techniques aimed at solving all problems, i.e., a "smart home" system. The method of construction of a mode of regulation of temperature with 
low consumption of energy in conditions of uncertainty in a finding of an optimum mode of rating values of parameters of thermal systems and maintenance of an economy effort of energy is developed. The structure of an energy-saving automatic control system was developed for thermal buildings under uncertain conditions. Coordinated operation of the various sensors that monitor the weather indoors, as well as the switches, control panels, and other devices can be applied automatically without human presence to ensure maximum comfort for all family members. The costs of the design and installation of the equipment will pay off within a short period of time by saving necessary resources.

\section{Conflict of Interest}

No potential conflict of interest relevant to this article was reported.

\section{Acknowledgements}

This paper was supported by the National Research Foundation of Korea (NRF) project "Intelligent Smart City Convergence Platform" (project number 20151D1A1A01061271).

\section{References}

[1] Y. N. Hassan, G. Ramanathan, and M. Kovatsch, "Demo: developing smart environments in the internet of things with the semantic IDE," in Proceedings of 2015 5th International Conference on the Internet of Things (IoT), Seoul, Korea, 2015, pp. 1-2.

[2] J. Jaykumar and A. Blessy, "Secure smart environment using IOT based on RFID," International Journal of Computer Science and Information Technologies, vol. 5, no. 2, pp. 2493-2496, 2014.

[3] A. Vimal Jerald, S. A. Rabara, and T. D. P. Bai, "Internet of things (IoT) based smart environment integrating various business applications," International Journal of Computer Applications, vol. 128, no. 8, pp. 32-37, 2015. https://doi.org/10.5120/ijca2015906622

[4] D. M. G. Preethichandra, "Design of a smart indoor air quality monitoring wireless sensor network for assisted living," in Proceedings of 2013 IEEE International Instrumentation and Measurement Technology Conference,
Minneapolis, MN, 2013. http://doi.org/10.1109/I2MTC. 2013.6555624

[5] T. Yu and C. Lin, "An intelligent wireless sensing and control system to improve indoor air quality: monitoring, prediction, and preaction" International Journal of Distributed Sensor Networks, vol. 2015, article no. 144, 2015. http://doi.org/10.1155/2015/140978

[6] S. Abraham and X. Li, "A cost-effective wireless sensor network system for indoor air quality monitoring applications," Procedia Computer Science, vol. 34, pp. 165-171, 2014. http://doi.org/10.1016/j.procs.2014.07.090

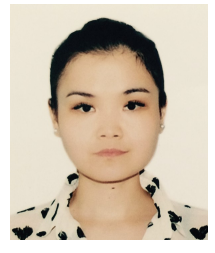

\begin{abstract}
Aigerim Bakatkaliyevna Altayeva received her B.S., M.Sc., from the Department of Computer Science, IITU, Kazakhstan, in 2012, 2014. Now she is a Ph.D. student at Gachon University. Her interesting part is AISmart
\end{abstract} City, Big data, Information retrieval etc.

Tel: +82-10-2992-1703

E-mail: aikosha1703@gmail.com

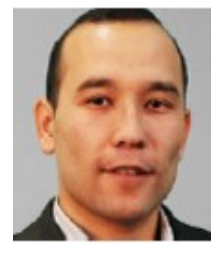

Batyrkhan Sultanovich Omarov received his B.S. and M.Sc. from Kazakh National University named after Al-Farabi, Almaty Kazakhstan, in 2008 and 2010, respectively. He is currently a Ph.D. student at The Universiti Tenaga National, Malaysia Kuala Lumpur. His research interests are image processing, computer vision, artificial intelligence, big data etc.

Tel: +82-10-2992-1703

E-mail: batyahan@gmail.com

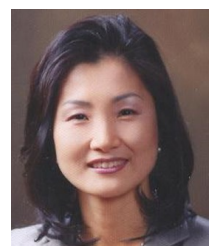

Young Im Cho received her B.S., M.Sc., and Ph.D. from the Department of Computer Science, Korea University, Korea, in 1988, 1990 and 1994, respectively. She is a professor at Gachon University. Her research interest includes AI, Big data, information retrieval, smart city etc.

Tel: +82-31-750-5800

Fax: +82-31-750-5662

E-mail: yicho@gachon.ac.kr 